JEFFREY L. MEYER

\title{
A reciprocity congruence for an analogue of the Dedekind sum and quadratic reciprocity
}

Journal de Théorie des Nombres de Bordeaux, tome 12, no 1 (2000), p. $93-101$

<http://www.numdam.org/item?id=JTNB_2000_12_1_93_0>

(C) Université Bordeaux 1, 2000, tous droits réservés.

L'accès aux archives de la revue «Journal de Théorie des Nombres de Bordeaux » (http://jtnb.cedram.org/) implique l'accord avec les conditions générales d'utilisation (http://www.numdam.org/conditions). Toute utilisation commerciale ou impression systématique est constitutive d'une infraction pénale. Toute copie ou impression de ce fichier doit contenir la présente mention de copyright.

\section{Numdam}

Article numérisé dans le cadre du programme

Numérisation de documents anciens mathématiques

http://www.numdam.org/ 


\title{
A Reciprocity Congruence for an Analogue of the Dedekind Sum and quadratic reciprocity
}

\author{
par JEFFREY L. MEYER
}

\begin{abstract}
RÉSUMÉ. Une loi de réciprocité est établie pour des sommes apparaissant dans les formules de transformations pour les logarithmes des fontions theta, sommes qui sont les analogues des sommes de Dedekind dans la transformation du logarithme de la fonction eta.

ABSTRACT In the transformation formulas for the logarithms of the classical theta-functions, certain sums arise that are analogous to the Dedekind sums in the transformation of the logarithm of the eta-function. A new reciprocity law is established for one of these analogous sums and then applied to prove the law of quadratic reciprocity.
\end{abstract}

\section{INTRODUCTION}

In Berndt [1] and Goldberg [2] the sum $S_{4}(d, c)$ is defined for $c>0$ by

$$
S_{4}(d, c)=\sum_{j=1}^{c-1}(-1)^{[d j / c]} .
$$

The sum is one of several involved in the multiplier systems for transformations of the logarithms of the classical theta-functions. We define two of them here. Let $q=e^{\pi i z}$ and for $\operatorname{Im}(z)>0$ define

$$
\theta_{3}(z)=\sum_{n=-\infty}^{\infty} q^{n^{2}} \text { and } \theta_{4}(z)=\sum_{n=-\infty}^{\infty}(-1)^{n} q^{n^{2}} .
$$

We prove a new reciprocity theorem for the sum $S_{4}(d, c)$. As an application of the theorem, we deduce the law of quadratic reciprocity.

Let $V=\left[\begin{array}{ll}a & b \\ c & d\end{array}\right]$ with $a d-b c=1$ and $V z=(a z+b) /(c z+d)$. We use the principal branch of the logarithm at all times. Berndt [1] proved that

Manuscrit reçu le 9 juin 1999. 
if $b$ is even, then

$$
\log \theta_{4}(V z)=\log \theta_{4}(z)+\frac{1}{2} \log (c z+d)-\frac{1}{4} \pi i-\frac{1}{4} \pi i S_{4}(d, c) .
$$

Goldberg [2] gives the following formula. If $a$ is even and $b, c$ and $d$ are odd, then

$$
\log \theta_{3}(V z)=\log \theta_{4}(z)+\frac{1}{2} \log (c z+d)-\frac{1}{4} \pi i-\frac{1}{4} \pi i S_{4}(d, c) .
$$

With the reciprocity theorem for the Dedekind sum $s(d, c)$ and connections between $s(d, c)$ and the Legendre-Jacobi symbol $\left(\frac{d}{c}\right)$, one can deduce the quadratic reciprocity law (See Rademacher and Grosswald [3],pp. 3435). Berndt [1] proved elegant reciprocity theorems for several sums that arise in the transformation formulas of the logarithms of the classical thetafunctions. None of these reciprocity theorems allow for both of the arguments in the sum to be odd. For the application of the sums arising in the theta-function transformations to quadratic reciprocity, however, we need a new reciprocity theorem where $c$ and $d$ are both odd. And, unlike the Dedekind sum, the sum $S_{4}(d, c)$ does not possess a reciprocity theorem. However, a reciprocity relation modulo 8 for the sum $S_{4}(d, c)$ does exist and this is sufficient to deduce the quadratic reciprocity theorem. For additional properties of $S_{4}(d, c)$ see [7] and [6].

To establish the connection between $S_{4}(d, c)$ and the Legendre-Jacobi symbol, we turn to Rademacher's book [8] (pp. 180-182) for the needed results. We use the same double subscript notation as Rademacher to state the theorem. For $\operatorname{Im}(z)>0$ and $\mu, \nu \in\{0,1\}$, let

$$
\theta_{\mu, \nu}(z)=\sum_{n=-\infty}^{\infty}(-1)^{\nu n} e^{(n+\mu / 2)^{2} \pi i z}
$$

Thus

$$
\theta_{0,0}(z)=\theta_{3}(z) \text { and } \theta_{0,1}(z)=\theta_{4}(z) .
$$

Note that we may allow integers other that 1 and 0 as subscripts since it can be shown that

$$
\theta_{\mu+2, \nu}(z)=(-1)^{\nu} \theta_{\mu, \nu}(z) \text { and } \theta_{\mu, \nu+2}(z)=\theta_{\mu, \nu}(z)
$$

We state one of Rademacher's transformation formulas.

Theorem 1. If $V=\left[\begin{array}{ll}a & b \\ c & d\end{array}\right]$ and $c$ is odd and positive, then

$$
\theta_{1+d, 1-b}(V z)=-i^{b} e^{\pi i b d / 4} \epsilon_{1} \sqrt{\frac{c z+d}{i}} \theta_{4}(z)
$$


where

$$
\epsilon_{1}=\epsilon_{1}(a, b, c, d)=\left(\frac{d}{c}\right) i^{(c-3) / 2} e^{(\pi i / 4) c(a+d)}
$$

\section{RECIPROCITY THEOREM FOR $S_{4}(d, c)$}

The next result is the foundation on which the new reciprocity theorem is based.

Theorem 2. Let $c$ and $d$ be positive, coprime, odd integers. Then

$$
S_{4}(c, d)+S_{4}(d, c)=-1+S_{4}\left(c^{2}, c d+1\right) .
$$

Proof. Choose $a$ and $b$ with $b>d, b$ even and $a d-b c=1$ and set

$$
V=\left[\begin{array}{ll}
a & b \\
c & d
\end{array}\right] \quad \text { and } \quad W=\left[\begin{array}{cc}
-d^{2} & c d-1 \\
c d+1 & -c^{2}
\end{array}\right]
$$

Then

$$
V W=\left[\begin{array}{cc}
b-d & c-a \\
d & -c
\end{array}\right]
$$

Note that in each matrix the upper right entry is even and the determinant is 1 . We use (1) with $V$ replaced with $V W$ to find that

(5) $\log \theta_{4}((V W) z)=\log \theta_{4}(z)+\frac{1}{2} \log (d z-c)-\frac{1}{4} \pi i-\frac{1}{4} \pi i S_{4}(-c, d)$.

Then we apply (1) with $z$ replaced by $W z$ to see that

(6) $\log \theta_{4}(V(W z))=\log \theta_{4}(W z)+\frac{1}{2} \log (c(W z)+d)$

$$
-\frac{1}{4} \pi i-\frac{1}{4} \pi i S_{4}(d, c)
$$

and finally we use (1) with $V$ replaced by $W$ to deduce that

(7) $\log \theta_{4}(W z)=\log \theta_{4}(z)+\frac{1}{2} \log \left((c d+1) z-c^{2}\right)$

$$
-\frac{1}{4} \pi i-\frac{1}{4} \pi i S_{4}\left(-c^{2}, c d+1\right) \text {. }
$$

We replace $\log \theta_{4}(W z)$ in (6) with (7) and then combine the result with (5) to conclude that

$$
-\frac{1}{4} \pi i S_{4}(-c, d)=-\frac{1}{4} \pi i S_{4}\left(-c^{2}, c d+1\right)-\frac{1}{4} \pi i-\frac{1}{4} \pi i S_{4}(d, c) .
$$

We have used the following lemma [5] to conclude that there are no branch changes with the logarithms, so the complete cancellation is justified. 
Lemma 1. Let $A, B, C$, and $D$ be real with $A$ and $B$ not both zero and $C>0$. Then for $\operatorname{Im}(z)>0$,

$$
\arg ((A z+B) /(C z+D))=\arg (A z+B)-\arg (C z+D)+2 \pi k,
$$

where $k$ is independent of $z$ and

$$
k= \begin{cases}1, & \text { if } A \leq 0 \text { and } A D-B C>0 \\ 0, & \text { otherwise. }\end{cases}
$$

Next we multiply (8) by $4 /(\pi i)$, rearrange, and use the fact that $S_{4}(-c, d)=-S_{4}(c, d)$ to conclude that

$$
S_{4}(c, d)+S_{4}(d, c)=-1+S_{4}\left(c^{2}, c d+1\right) .
$$

The symmetry between $c$ and $d$ on the left-hand side of the equation in Theorem 3 leads immediately to the next result.

Corollary 1. If $c$ and $d$ are coprime, odd, positive integers, then

$$
S_{4}\left(c^{2}, c d+1\right)=S_{4}\left(d^{2}, c d+1\right) .
$$

The final step towards the desired reciprocity relation is the following congruence relation.

Lemma 2. Let $d$ be an odd prime and $c>d$ be an odd, positive integer coprime to $d$. Then

$$
S_{4}\left(d^{2}, c d+1\right) \equiv c d \quad(\bmod 8) .
$$

Proof. Using the definition of $S_{4}(d, c)$ and the fact that

$$
[x]-2\left[\frac{x}{2}\right]= \begin{cases}0, & \text { if }[x] \text { is even, } \\ 1, & \text { if }[x] \text { is odd }\end{cases}
$$

we see that

$$
\text { (9) } \begin{aligned}
S_{4}\left(d^{2}, c d+1\right) & =\sum_{j=1}^{c d}(-1)\left[\frac{j d^{2}}{c d+1}\right] \\
& =\#\left\{\left[j d^{2} /(c d+1)\right] \text { even }\right\}-\#\left\{\left[j d^{2} /(c d+1)\right] \text { odd }\right\} \\
& =c d-2 \sum_{j=1}^{c d}\left[\frac{j d^{2}}{c d+1}\right]+4 \sum_{j=1}^{c d}\left[\frac{j d^{2}}{2(c d+1)}\right] .
\end{aligned}
$$

If $m$ and $n$ are positive and coprime integers, recall that (see [4] p. 186, for example)

$$
\sum_{j=1}^{n-1}\left[\frac{m j}{n}\right]=\frac{(n-1)(m-1)}{2}
$$


We now apply (10) to the first of the final two sums in (9) and separate the terms in the second sum on the right according to the parity of $j$. Thus (9) becomes

$$
\begin{aligned}
S_{4}\left(d^{2}, c d+1\right)=c d-\left(d^{2}-1\right) c d & +4 \sum_{j=1}^{(c d-1) / 2}\left[\frac{j d^{2}}{c d+1}\right] \\
& +4 \sum_{j=1}^{(c d+1) / 2}\left[\frac{j d^{2}}{c d+1}-\frac{d^{2}}{2(c d+1)}\right]
\end{aligned}
$$

Let

$$
N=\#\left\{j=1,2, \ldots, \frac{c d+1}{2}:\left\{\frac{j d^{2}}{c d+1}\right\}<\frac{d^{2}}{2(c d+1)}\right\},
$$

where $\{x\}=x-[x]$ denotes the fractional part of $x$. The final sum in (11) can be simplified since $d<c$ and $\left[d^{2} / 2\right]=\left(d^{2}-1\right) / 2$ for $d$ odd. Note that

$$
\sum_{j=1}^{(c d+1) / 2}\left[\frac{j d^{2}}{c d+1}-\frac{d^{2}}{2(c d+1)}\right]=\sum_{j=1}^{(c d-1) / 2}\left[\frac{j d^{2}}{c d+1}\right]+\frac{d^{2}-1}{2}-N .
$$

We conclude from (11) and (13) that

$$
S_{4}\left(d^{2}, c d+1\right)=c d-\left(d^{2}-1\right) c d+8 \sum_{j=1}^{(c d-1) / 2}\left[\frac{j d^{2}}{c d+1}\right]+2\left(d^{2}-1\right)-4 N .
$$

Since $d$ is odd and thus $d^{2} \equiv 1(\bmod 8)$, we deduce the congruence

$$
S_{4}\left(d^{2}, c d+1\right) \equiv c d+4 N \quad(\bmod 8) .
$$

We claim that $N$ is even. Let

$$
n=\#\left\{k=1,2, \ldots, \frac{d^{2}-1}{2}: \operatorname{LPR}_{d^{2}}(k(c d+1))>\frac{d^{2}}{2}\right\},
$$

where $L P R_{l}(m)$ denotes the least positive residue of $m$ modulo the positive integer $l$. Observe that

$$
\left\{\frac{j d^{2}}{c d+1}\right\}<\frac{d^{2}}{2(c d+1)}
$$

if and only if there exists a positive integer $k$ such that

$$
2 k(c d+1)<2 j d^{2}<2 k(c d+1)+d^{2} .
$$

We rewrite (16) in the form

$$
\frac{k(c d+1)}{d^{2}}<j<\frac{k(c d+1)}{d^{2}}+\frac{1}{2} .
$$


Since the interval $\left(k(c d+1) / d^{2}, k(c d+1) / d^{2}+1 / 2\right)$ has length $1 / 2$, it contains an integer $j$ if and only if $k$ satisfies

$$
\left\{\frac{k(c d+1)}{d^{2}}\right\}>\frac{1}{2}
$$

or, in other words, if and only if $L P R_{d^{2}}(k(c d+1))>d^{2} / 2$. Thus $N=n$.

The final claim in the proof of the congruence is the following: The number $n$ defined above is even.

The proof of this claim is a careful application of the method used in the standard proof of Gauss's Lemma (see [4], p. 133 , for example).

Let $r_{1}, r_{2}, \ldots, r_{n}$ be the $n$ residues of $k(c d+1), 1 \leq k \leq\left(d^{2}-1\right) / 2$, falling in the upper half of the least positive residue system $\left(\bmod d^{2}\right)$ and let $s_{1}, s_{2}, \ldots, s_{l}$ be those in the lower half. Then $n+l=\left(d^{2}-1\right) / 2$. Next we consider $r_{i}^{\prime}=d^{2}-r_{i}$ for $i=1,2, \ldots, n$. Each of the $r_{i}^{\prime}$ are distinct and we can say further that no $r_{i}^{\prime}=s_{j}$ for any $i$ and $j$.

Note that there are $\left[\left(d^{2}-1\right) /(2 d)\right]=(d-1) / 2$ positive multiples of $d$ that are less than $\left(d^{2}-1\right) / 2$. Now for $k=m d, 1 \leq m \leq(d-1) / 2$,

$$
k(c d+1)=m d(c d+1) \equiv m d \quad\left(\bmod d^{2}\right) .
$$

From (18), we conclude that

$$
L P R_{d^{2}}(m d(c d+1))=m d<\frac{d^{2}}{2} .
$$

Thus all of the residues produced when $k$ is a multiple of $d$ are among the $s_{j}$ and are in fact the positive multiples of $d$ that are less than $d^{2} / 2$. We remove them from the list $\left\{s_{1}, \ldots, s_{l}\right\}$, reindex this set and put

$$
l^{\prime}=l-\frac{d-1}{2} \text {. }
$$

So now we consider the $n r_{i}$ 's, and the $l^{\prime} s_{j}$ 's, with $n+l^{\prime}=d(d-1) / 2$ residues altogether. By the distinctness of the $r_{i}^{\prime}$, we conclude that the $r_{i}^{\prime}$ and the $s_{j}$ are some rearrangement of the numbers $1,2, \ldots,\left(d^{2}-1\right) / 2$ with the multiples of $d$ removed. Thus

$$
r_{1}^{\prime} r_{2}^{\prime} \cdots r_{n}^{\prime} s_{1} s_{2} \cdots s_{l^{\prime}}=\prod_{\substack{1 \leq \alpha \leq\left(d^{2}-1\right) / 2 \\(d, \alpha)=1}} \alpha
$$

or, by definition of the $r_{i}^{\prime}$,

$$
\left(d^{2}-r_{1}\right)\left(d^{2}-r_{2}\right) \cdots\left(d^{2}-r_{n}\right) s_{1} s_{2} \cdots s_{l^{\prime}}=\prod_{\substack{1 \leq \alpha \leq\left(d^{2}-1\right) / 2 \\(d, \alpha)=1}} \alpha
$$


yielding the congruence

$$
(-1)^{n} r_{1} r_{2} \cdots r_{n} s_{1} s_{2} \cdots s_{l^{\prime}} \equiv \prod_{\substack{1 \leq \alpha \leq\left(d^{2}-1\right) / 2 \\(d, \alpha)=1}}^{\alpha} \quad\left(\bmod d^{2}\right)
$$

Now we rewrite (19) with the $r_{i}$ and the $s_{j}$ in their original form, with some possible rearrangement, to get

$$
(-1)^{n}(c d+1) \cdot 2(c d+1) \cdots \frac{d^{2}-1}{2}(c d+1) \equiv \prod_{\substack{1 \leq \alpha \leq\left(d^{2}-1\right) / 2 \\(d, \alpha)=1}} \alpha \quad\left(\bmod d^{2}\right) .
$$

Recall the exclusion of the multiples of $d$ on each side. Because $d$ is prime, we may cancel the common factors from each side of the congruence (20) and conclude that

$$
(c d+1)^{d(d-1) / 2} \equiv(-1)^{n} \quad\left(\bmod d^{2}\right) .
$$

But by the binomial theorem,

$$
(c d+1)^{d(d-1) / 2} \equiv 1+\frac{d(d-1)}{2} c d \equiv 1 \quad\left(\bmod d^{2}\right) .
$$

From (21) and (22), we deduce that $n$ is even and the proof of the claim is complete. And thus, from (14), we have

$$
S_{4}\left(d^{2}, c d+1\right) \equiv c d \quad(\bmod 8)
$$

We now assemble Theorem 3, Corollary 1, and Lemma 2 to reach our reciprocity result.

Theorem 3. Let $d$ be an odd prime and $c>d$ be an odd, positive integer coprime to $d$. Then

$$
S_{4}(c, d)+S_{4}(d, c) \equiv-1+c d \quad(\bmod 8) .
$$

\section{The LAW OF QUADRATIC RECIPROCITY}

As an application of Theorem 4, we offer a new proof of the law of quadratic reciprocity.

Theorem 4. Let $c$ and $d$ be distinct odd primes. Then

$$
\left(\frac{c}{d}\right)\left(\frac{d}{c}\right)=(-1)^{\frac{c-1}{2} \frac{d-1}{2}} \text {. }
$$


Proof. Given $c$ and $d$, there are integers $a$ and $b$ with $b$ even such that $a d-b c=1$. Let $V=\left[\begin{array}{ll}a & b \\ c & d\end{array}\right]$ and $W=\left[\begin{array}{cc}-b & -a \\ d & c\end{array}\right]$. Note that $a$ is necessarily odd. From (3), (4) and recalling that $a, c$, and $d$ are odd and $b$ is even, we see that

$$
\begin{aligned}
\theta_{1+d, 1-b}(V z) & =\theta_{1+d, 1}(V z) \\
& =(-1)^{(d+1) / 2} \theta_{0,1}(V z) \\
& =(-1)^{(d+1) / 2} \theta_{4}(V z)
\end{aligned}
$$

and

$$
\begin{aligned}
\theta_{1+c, 1+a}(W z) & =\theta_{1+c, 0}(W z) \\
& =\theta_{0,0}(W z) \\
& =\theta_{3}(W z) .
\end{aligned}
$$

From Theorem 1, (23) and (24), we have

$$
\begin{aligned}
& \theta_{4}(V z)=(-1)^{(-d-1) / 2}\left(-i^{b}\right) e^{\pi i b d / 4} \\
& \times\left(\frac{d}{c}\right) i^{(c-3) / 2} e^{\pi i(a c+d c) / 4} \sqrt{\frac{c z+d}{i}} \theta_{4}(z)
\end{aligned}
$$

and

$$
\theta_{3}(W z)=-i^{-a} e^{-\pi i a c / 4}\left(\frac{c}{d}\right) i^{(d-3) / 2} e^{\pi i(d c-b d) / 4} \sqrt{\frac{d z+c}{i}} \theta_{4}(z) .
$$

We also have, from (1) and (2),

$$
\log \theta_{4}(V z)=\log \theta_{4}(z)+\frac{1}{2} \log (c z+d)-\frac{1}{4} \pi i-\frac{1}{4} \pi i S_{4}(d, c)
$$

and

$$
\log \theta_{3}(W z)=\log \theta_{4}(z)+\frac{1}{2} \log (d z+c)-\frac{1}{4} \pi i-\frac{1}{4} \pi i S_{4}(c, d) .
$$

Next, we multiply (25) and (26) and partially simplify the result to deduce that

$$
\begin{aligned}
& \theta_{4}(V z) \theta_{3}(W z)=(-1)^{(-d-1) / 2} i^{b-a} i^{(c+d-6) / 2} e^{\pi i d c / 2} i^{-1} \\
& \times\left(\frac{c}{d}\right)\left(\frac{d}{c}\right) \sqrt{c z+d} \sqrt{d z+c} \theta_{4}(z)^{2} .
\end{aligned}
$$

We exponentiate and then multiply (27) and (28) to see that

(30) $\theta_{4}(V z) \theta_{3}(W z)=\theta_{4}(z)^{2} \sqrt{c z+d} \sqrt{d z+c} e^{-\pi i / 2}$

$$
\times e^{-(\pi i / 4)\left(S_{4}(c, d)+S_{4}(d, c)\right)} .
$$


From (29), (30) and Lemma 1, we deduce that

$$
\begin{aligned}
(-1)^{(-d-1) / 2} i^{(2 b-2 a+c+d-6) / 2} e^{\pi i d c / 2} i^{-1}\left(\frac{c}{d}\right)\left(\frac{d}{c}\right) & \\
= & e^{-\pi i / 2} e^{-(\pi i / 4)\left(S_{4}(c, d)+S_{4}(d, c)\right) .}
\end{aligned}
$$

Next, we simplify (31) to find that

$$
e^{(\pi i / 4)(-2 a+2 b+c-d+2 c d)}\left(\frac{c}{d}\right)\left(\frac{d}{c}\right)=e^{-(\pi i / 4)\left(S_{4}(c, d)+S_{4}(d, c)\right)}
$$

Note that since $d$ is odd and $a d-b c=1$, we have that $a \equiv d+d b c(\bmod 8)$. From this fact and the application of Theorem 3 to (32), we conclude that

$$
\left(\frac{c}{d}\right)\left(\frac{d}{c}\right)=e^{(\pi i / 4)(3 d-c-2 b+2 b c d-3 c d+1)} .
$$

A straight-forward calculation shows that

$$
3 d-c-2 b+2 b c d-3 c d+1 \equiv(c-1)(d-1) \quad(\bmod 8) .
$$

Using (34) in (33), we deduce that

$$
\left(\frac{c}{d}\right)\left(\frac{d}{c}\right)=e^{(\pi i / 4)(c-1)(d-1)}=(-1)^{\frac{c-1}{2} \frac{d-1}{2}}
$$

as desired.

\section{REFERENCES}

[1] B.C. Berndt, Analytic Eisenstein series, theta-functions, and series relations in the spirit of Ramanujan. J. Reine Angew. Math. 303/304 (1978), 332-365.

[2] L.A. Goldberg, Transformations of theta-functions and analogues of dedekind sums. Ph.D. thesis, Thesis, University of Ilinois, Urbana, 1981.

[3] H. Rademacher E. Grosswald, Dedekind sums. Carus Math. Monogr., vol. 16, Mathematical Association of America, Washington, D.C., 1972.

[4] H. Montgomery I. Niven, H. Zuckerman, An introduction to the theory of numbers. 5th ed., John Wiley and Sons, New York, 1991.

[5] J. Lewittes, Analytic continuation of Eisenstein series. Trans. Amer. Math. Soc. 171 (1972), 469-490.

[6] J.L. Meyer, Analogues of dedekind sums. Ph.D. thesis, University of Mlinois, Urbana, 1997.

[7] _ Properties of certain integer-valued analogues of Dedekind sums. Acta Arithmetica 82 (1997), 229-242.

[8] H. Rademacher, Topics in analytic number theory. Springer-Verlag, New York, 1973.

Jeffrey L. MEYER

15 Carnegie Hall

Department of Mathematics

Syracuse University

Syracuse, NY 13244, USA

E-mail : jlmeye010mailbox.syr.edu 